\title{
OPEN WHO grade I meningiomas that show regrowth after gamma knife radiosurgery often show 1 p36 loss
}

\author{
Pim J. J. Damen ${ }^{1,5}$, Vincent J. Bulthuis ${ }^{2,5}$, Patrick E. J. Hanssens ${ }^{3}$, Suan Te Lie ${ }^{3}$, \\ Ruth Fleischeuer ${ }^{4}$, Veerle Melotte ${ }^{1}$, Kim A. Wouters ${ }^{1}$, Andrea Ruland ${ }^{1}$, \\ Jan Beckervordersandforth ${ }^{1}$ \& Ernst Jan M. Speel ${ }^{1 \bowtie}$
}

WHO grade I meningiomas occasionally show regrowth after radiosurgical treatment, which cannot be predicted by clinical features. There is increasing evidence that certain biomarkers are associated with regrowth of meningiomas. The aim of this retrospective study was to asses if these biomarkers could be of value to predict regrowth of WHO grade I meningiomas after additive radiosurgery. Forty-four patients with WHO grade I meningiomas who underwent additive radiosurgical treatment between 2002 and 2015 after Simpson IV resection were included in this study, of which 8 showed regrowth. Median follow-up time was 64 months (range 24-137 months). Tumors were analyzed for the proliferation marker Ki-67 by immunohistochemistry and for deletion of $1 \mathrm{p} 36$ by fluorescence in situ hybridization (FISH). Furthermore, genomic DNA was analyzed for promoter hypermethylation of the genes NDRG1-4, SFRP1, HOXA9 and MGMT. Comparison of meningiomas with and without regrowth after radiosurgery revealed that loss of $1 p 36(p=0.001)$ and hypermethylation of NDRG1 $(p=0.046)$ were correlated with regrowth free survival. Loss of $1 p 36$ was the only parameter that was significantly associated with meningioma regrowth after multivariate analysis $(p=0.01)$. Assessment of 1 p36 loss in tumor tissue prior to radiosurgery might be considered an indicator of prognosis/ regrowth. However, this finding has to be validated in an independent larger set of tumors.

Meningiomas are brain tumors originating from the arachnoid cap cells of the arachnoid villi. They account for more than one third of all primary central nervous system neoplasms and are the second most common brain tumors ${ }^{1}$. Meningiomas are histologically classified according to the World Health Organization (WHO) into a benign, slowly growing WHO grade I $(\sim 80 \%)$ and the more invasive and aggressive atypical grade II $(\sim 18 \%)$ and anaplastic grade III ( 2\%) group ${ }^{2}$.

Generally, asymptomatic meningiomas are monitored regular follow up. When the tumor becomes symptomatic or shows radiological progression treatment can be indicated. The primary treatment of symptomatic meningiomas is surgery. Dependent on the completeness of resection, which can be indicated by Simpson grade I-IV, the 5-years recurrence free survival rate decreases from 98 to $57 \%$ respectively ${ }^{3}$. In patients with high surgical risk or tumors in eloquent or inaccessible regions either conventional or stereotactic radiotherapy is a viable alternative. ${ }^{4}$ Gamma Knife Radiosurgery (GKRS) is one of the most frequently used type of radiosurgery in meningiomas. The 5-year tumor control rate after GKRS is $95 \%$ in WHO grade I, $50 \%$ in grade II and $20 \%$ in grade III tumors ${ }^{5}$. Despite the fact that most WHO grade I meningiomas can be controlled well, a small group shows regrowth after radiosurgery, which is difficult to predict by histopathology on the tissue biopsy prior to treatment. One possibility would be that these tumors might present atypical morphological features which have been previously associated with a worse outcome ${ }^{6}$. Thus, it is crucial to analyse if such morphological features or other features such as molecular alterations can be found in WHO I tumors that show regrowth after radiosurgery.

\footnotetext{
${ }^{1}$ Department of Pathology, GROW School for Oncology and Developmental Biology, Maastricht University Medical Centre, P. Debyelaan 25, Postbox 5800, 6202 AZ Maastricht, The Netherlands. ${ }^{2}$ Department of Neurosurgery, Maastricht University Medical Center, Maastricht, The Netherlands. ${ }^{3}$ Gamma Knife Center Tilburg, ETZ-Elisabeth Hospital, Tilburg, The Netherlands. ${ }^{4}$ Department of Pathology, ETZ-Elisabeth Hospital, Tilburg, The Netherlands. ${ }^{5}$ These authors contributed equally: Pim J. J. Damen and Vincent J. Bulthuis. ${ }^{\varpi}$ email: ernstjan.speel@ mumc.nl
} 
Several studies have been conducted to investigate the molecular mechanisms underlying meningioma tumorigenesis, progression and recurrence. Some studies show that proliferation marker Ki-67 correlates with significantly shorter recurrence-free survival (RFS) in meningiomas of all grades, if for example tumors have a proliferation index $>3 \%^{7}$ or when $>25$ positive cell nuclei per square millimetre ${ }^{8}$ are observed. Deletion of chromosome loci have been associated with meningioma recurrence, with $1 \mathrm{p} 36$ occurring most frequently. A study of 36 meningiomas ( $59 \%$ WHO 1) showed that tumors with 1 p36 loss had a higher recurrence rate ( $25.9 \%$ vs. $11.5 \%$, $p=0.003)^{9}$ and another study of 149 WHO 1 meningiomas showed that 1 p36 loss correlated with a shorter RFS at $2.5,5$ and 10 years $(p=0.008, p=0.009 \text { and } p=0.01 \text { respectively })^{10}$. The $\mathrm{N}$-myc Downstream Regulated Gene (NDRG)-family has also been related to central nervous system (CNS)-tumors, including meningioma. Of the 4 family members, the NDRG2 mRNA and protein expression levels were found to be reduced in higher grade and clinically aggressive meningiomas, which was associated with hypermethylation of the NDRG2 promotor $^{11}$.

In addition, a tree-fold reduction in NDRG2 mRNA levels was reported when comparing primary with recurrent tumors $(p=0.009)^{12}$. In contrast, $N D R G 4$ has been shown to be overexpressed in high grade malignant meningioma cell lines. Although it is unknown how this overexpression is induced, silencing of NDRG4 inhibited proliferation and induced apoptotic cell death ${ }^{13,14}$. A couple of other studies have reported silencing of gene expression by promotor hypermethylation to play a role in meningioma tumorigenesis. In a mRNA profiling study SFRP1 was one of the genes which expression was downregulated in recurrent meningiomas $(p=0.02)^{15,16}$. In a genome-wide methylation analysis HOXA9 was correlated with recurrence ${ }^{17}$. This was also found in an independent study analysing $\operatorname{HOXA7,9}$ and $10(p=0.0019)^{18}$. Finally, promotor hypermethylation of $\mathrm{O}^{6}$-methylguanine-DNA methyltransferase $(M G M T)$ has been found in $0-22 \%$ in meningiomas and grade II meningiomas showed twofold higher percentage of MGMT promotor methylation than grade I tumor ${ }^{19-21}$.

The objective of this study was to investigate if the above-mentioned markers also can be used to recognise WHO grade I meningiomas that show regrowth after GKRS. Treatment for these potentially more aggressive tumors could be adapted.

For this purpose, 44 WHO grade I meningiomas, including 8 showing regrowth after radiosurgery, were analysed for Ki-67 and NDRG4 protein expression, loss of $1 \mathrm{p} 36$ and promotor hypermethylation of the genes NDRG1-4, SFRP1, HOXA9 and MGMT.

\section{Material and methods}

A detailed description of materials and methods can be found in Supplementary "Methods".

Patient and tissue samples. Samples were collected from a series of $\sim 600$ meningiomas treated in ETZElisabeth Hospital (Tilburg, Netherlands) with GKRS between 2002 and 2015. For this study we selected all WHO grade I meningiomas which were partially resected (Simpson grade IV; $\mathrm{n}=44$ ) and received additive GKRS. The median time between resection and additive GKRS was 7 months (range 1-13 months), with no proven regrowth within this period of time. Of the 44 meningiomas included, 8 tumors showed regrowth.

Tumor material collected at primary surgery was retrieved from 5 different Dutch hospitals. Patients were followed with a median follow-up of 64 months (range 24-137 months; regrowth group: 50 months [range 29-81 months]; no regrowth group: 73 months [range 23-137 months]). All regrowth was in-field. Histologically, 6 meningioma subgroups were classified. Table 1 summarizes clinical and histopathological parameters of the 44 meningiomas.

This study was approved by the Medical Ethical Committee (METC 154-098, MUMC Maastricht). Patient material was used according to the Code for Proper Secondary Use of Human Tissue (Federation of Medical Scientific Societies, The Netherlands; 2013).

Radiotherapy. Treatment planning for single session GKRS using Leksell Gamma Knife 4C or Perfexion (Elekta AB, Stockholm, Sweden) was performed with Leksell Gamma Plan (Elekta AB) based on high resolution Gadolinium enhanced stereotactic planning T1-weighted MRI scans with G-frame. The target volume was defined as the contrast-enhancing lesion. Only part of the dural tail adjacent to the tumor was included in the target volume planning ${ }^{22}$. A median dose of $11 \mathrm{~Gy}(10-18 \mathrm{~Gy}$ ) was prescribed to that isodose-line covering $90-100 \%$ of the target volume, resulting in a median marginal dose of $11 \mathrm{~Gy}(10-17.8 \mathrm{~Gy})$.

Follow-up imaging was carried out at 6 months after radiosurgery, followed by a 1-year interval in the next 3 years. Tumor growth was defined as a minimum diameter increase of $2 \mathrm{~mm}$ in any direction. Tumor growth was considered an out-field increase of tumor volume in the axial planes. If growth was observed inside the treated volume it was classified as in-field regrowth, whereas growth outside the treatment volume was considered out-field regrowth.

Immunohistochemistry. Immunohistochemistry on $3 \mu \mathrm{m}$-thick formalin-fixed, paraffin-embedded (FFPE) tissue sections using primary antibodies directed against Ki-67 (MIB-1) and NDRG4, was performed using the DAKO Autostainer Link 48, according to standard protocols. MIB-1 nuclear staining was subdivided in 2 categories: $\leq 1 \%$ and $>1 \%$. For NDRG4 staining the intensity $(0-3+)$ and percentage for both nuclear and/or cytoplasmic stained tumor cells were evaluated.

Fluorescence in situ hybridization (FISH). FISH was performed on $3 \mu \mathrm{m}$-thick FFPE tissue sections, using a 1p36/1q25 specific probe mixture, as previously described ${ }^{23}$. At least 20 non-overlapping nuclei per sample were scored for evaluation, using a Leica DM5000b fluorescence microscope, with appropriate fluorochrome filter sets. 1p36 deletion was indicated if the ratio between 1p36/1q25 was less than 0.8 . 


\begin{tabular}{|c|c|c|c|c|c|}
\hline & No regrowth & Regrowth & Total & Fisher's exact ( $p$ value) & Kaplan-Meier ( $p$ value) \\
\hline No of patients & 36 & 8 & 44 & & \\
\hline Age (yrs) & & & & 0.26 & 0.124 \\
\hline$<58$ & $22(88 \%)$ & $3(12 \%)$ & 25 & & \\
\hline$\geq 58$ & $14(74 \%)$ & $5(26 \%)$ & 19 & & \\
\hline Gender & & & & 0.67 & 0.161 \\
\hline Female & $26(84 \%)$ & $5(16 \%)$ & 31 & & \\
\hline Male & $10(77 \%)$ & $3(23 \%)$ & 13 & & \\
\hline Tumor localization & & & & 0.11 & 0.056 \\
\hline Inside skull base & $17(94 \%)$ & $1(6 \%)$ & 18 & & \\
\hline (Petro)Clival & 2 & 0 & 2 & & \\
\hline Cavernous Sinus & 4 & 0 & 4 & & \\
\hline Frontobasal & 1 & 0 & 1 & & \\
\hline Parasellar & 2 & 0 & 2 & & \\
\hline Petrous Bone & 2 & 0 & 2 & & \\
\hline Planum Sphenoidale & 1 & 0 & 1 & & \\
\hline Sella & 0 & 1 & 1 & & \\
\hline Sphenoid Wing & 1 & 0 & 1 & & \\
\hline Cerebral Pontine Angel & 4 & 0 & 4 & & \\
\hline Outside skull base & $19(73 \%)$ & $7(27 \%)$ & 26 & & \\
\hline Convexity & 4 & 2 & 6 & & \\
\hline Falcine & 5 & 2 & 7 & & \\
\hline Orbital & 1 & 0 & 1 & & \\
\hline Parasagital & 0 & 1 & 1 & & \\
\hline Posterior Fossa & 3 & 1 & 4 & & \\
\hline Tentorial & 5 & 0 & 5 & & \\
\hline Tentorial Notch & 1 & 1 & 2 & & \\
\hline Tumor volume $\left(\mathrm{cm}^{3}\right)$ & & & & $0.69^{* *}$ & 0.559 \\
\hline 0 to 5 & 23 & 4 & 27 & & \\
\hline 5 to 10 & 9 & 2 & 11 & & \\
\hline 10 to 15 & 4 & 2 & 6 & & \\
\hline Dose $100 \%$ & & & & 1 & 0.663 \\
\hline$<11 \mathrm{~Gy}$ & $9(82 \%)$ & $2(18 \%)$ & 11 & & \\
\hline$\geq 11 \mathrm{~Gy}$ & $27(82 \%)$ & $6(18 \%)$ & 33 & & \\
\hline Prescribed dose & & & & 1 & 0.565 \\
\hline$<13 \mathrm{~Gy}$ & $8(80 \%)$ & $2(20 \%)$ & 10 & & \\
\hline$\geq 13 \mathrm{~Gy}$ & $28(82 \%)$ & $6(18 \%)$ & 34 & & \\
\hline Histotype & & & & 0.45 & 0.211 \\
\hline Meningothelial & $21(78 \%)$ & $6(22 \%)$ & 27 & & \\
\hline Not meningothelial & $15(88 \%)$ & $2(12 \%)$ & 17 & & \\
\hline Fibrous & 4 & 0 & 4 & & \\
\hline Transitional & 8 & 1 & 9 & & \\
\hline Angiomatous & 1 & 0 & 1 & & \\
\hline Psammomatous & 2 & 0 & 2 & & \\
\hline Microcystic & 0 & 1 & 1 & & \\
\hline
\end{tabular}

Table 1. Clinical, radiobiological and histopathological parameters in relation to regrowth of irradiated WHO grade I meningiomas. ${ }^{*}$ After combining tumor volume groups ' $5-10 \mathrm{~cm}^{3}$ ' and ' $10-15 \mathrm{~cm}^{3}$.

DNA isolation from tumor tissue. DNA was isolated using the Promega Maxwell 16 FFPE plus LEV DNA purification kit according to manufacturer's instructions.

Methylation-specific polymerase chain reaction (MSP). The methylation status of NDRG1-4, MGMT, SFRP1, HOXA9 and MGMT was assessed by MSP. 500 ng DNA was modified by sodium bisulphite, using the Zymo Research EZ DNA Methylation kit, according to manufacturer's instructions. Primer sequences and corresponding annealing temperatures are summarised in Supplementary Table S1. The PCR products were analysed on a $2 \%$ agarose gel. 
Statistical analysis. Statistical analysis was processed with the statistical package for the social sciences (SPSS, version 23 Chicago, IL) computer software for Windows. Fisher's Exact test was used for correlating parameters with regrowth. Kaplan-Meier's Log-Rank test was used for univariate analysis of progression free survival. Multivariate analysis was performed using Cox Regression Model. Statistical significance was presumed as $p<0.05$.

Ethics approval and consent to participate. This study was approved by the Medical Ethical Committee (METC 15-4-098, MUMC Maastricht). Patient material was used according to the Code for Proper Secondary Use of Human Tissue (Federation of Medical Scientific Societies, The Netherlands; 2013).

Conference presentation. This study was presented in part orally at the Leksell Gamma Knife Society Meeting, Amsterdam, May 15-19, 2016. ${ }^{24}$

\section{Results}

A total of 44 meningiomas from 44 patients were included in this study, of which 8 (18\%) showed regrowth during follow-up after a median time of 51 months. Local control rate was $100 \%$ after 2 years and $89 \%$ after 5 years. In all cases, the regrowth occurred infield and no marginal regrowth was observed. Clinical, radiobiological and histopathological parameters, including tumor volume and radiotherapy dose, in relation to regrowth can be found in Table 1 . These parameters did not show significant association with regrowth.

FISH for 1p36/1q25 was performed on 43 meningioma samples and 2 nontumoral brain tissue control samples (Fig. 1A-B). One meningioma sample did not have enough tissue for FISH analysis. Deletion of chromosome 1 p36 was detected in 8 meningiomas. The nontumoral brain tissue control samples showed disomy for both $1 \mathrm{p} 36$ and 1q25. Five out of 8 (63\%) meningiomas with chromosome 1p36 deletion showed regrowth, compared to 3 out of $32(9 \%)$ samples without deletion of $1 \mathrm{p} 36(p=0.003)$. Chromosome 1p36 loss was correlated with regrowth $(p=0.003)$ as well as regrowth free survival $(p=0.001)$, as seen in Table 2 and Fig. 2.

Nuclear Ki-67 expression was examined in 44 meningioma samples and 2 nontumoral brain tissue control samples using MIB-1 immunostaining (Fig. 1C-D). Twenty-three meningiomas had a proliferation index $\leq 1.0 \%$, of which $4(17 \%)$ showed regrowth, compared to $19(83 \%)$ showing no regrowth. Twenty-one meningiomas had labelling $>1.0 \%$, of which $4(19 \%)$ showed regrowth, compared to $17(81 \%)$ non-regrowth. Ki-67 proliferation index did not correlate with survival.

MSP for NDRG1-4, SFRP1, HOXA9 and MGMT was performed on 41 meningioma samples (see Table 2 and Fig. 1G-H) and 10 nontumoral control brain tissue samples. From 3 meningioma samples too less DNA could be isolated for MSP analysis. No hypermethylation was found for NDRG3 and NDRG4, and NDRG4 protein expression could not be detected by immunohistochemistry (Fig. 1E-H). The promotor regions of NDRG1 and 2 were hypermethylated in 5 samples of which $40 \%$ showed regrowth, compared to $14 \%$ in the non-hypermethylated group. For MGMT this was $20 \%$ vs. $15 \%$, for HOXA9 $18 \%$ vs. $11 \%$ and for SFRP1 $14 \%$ vs. $18 \%$, respectively. None of 10 nontumoral control samples showed hypermethylation for any of the genes analysed. Only hypermethylation of NDRG1 was correlated with regrowth free survival $(p=0.046)$.

Multivariate Cox Regression analysis was performed for all parameters which were significant $(\mathrm{p}<0.05)$ on univariate analysis of regrowth free survival (i.e. deletion $1 \mathrm{p} 36$ and methylation of NDRG1). Deletion of 1p36 kept its significance after multivariate analysis $(p=0.01)$.

\section{Discussion}

WHO grade I meningioma patients who undergo additive radiosurgical treatment after Simpson grade IV resection sometimes show tumor regrowth. The aim of this retrospective study was to asses if biomarkers may be of value to predict regrowth of WHO grade I meningiomas after additive radiosurgery. Treatment for these potentially more aggressive tumors then could be adapted.

From a series of 44 of these cases, treated in a Dutch GKRS center in the period of 2002-2015, 8 patients showed regrowth. We found loss of chromosome 1 p36 and to a lesser extent hypermethylation of NDRG1 to be correlated with regrowth free survival, while clinical, radiobiological and histopathological parameters, expression of Ki-67 and NDRG4 and hypermethylation of NDRG2-4, SFRP1, HOXA9 and MGMT were not.

Chromosome $1 \mathrm{p}$ loss is the second most common abnormality in meningioma and is more frequently detected in higher grade tumors. Loss of $1 \mathrm{p}$ has been found in 13-26\% of Grade I, $40-76 \%$ of Grade II and $70-100 \%$ of Grade III meningiomas ${ }^{25-27}$. Loss of $1 p$, along with $9 p$ and $14 q$, have been identified more often in high grade meningiomas which show higher rate of recurrence ${ }^{28}$. Interestingly, Grade I meningiomas showing recurrence had significantly more chromosome copy number alterations with a mean of $19 \%$ of genome disrupted, including $1 \mathrm{p}$ deletion, compared to $3 \%$ in low-grade samples not showing recurrence ${ }^{29}$. Tabernero et al. found that the common deleted region of $1 \mathrm{p}$ was pter $\rightarrow 1 \mathrm{p} 34.2^{30}$, which contains the $1 \mathrm{p} 36$ locus evaluated in our present study. A gene mapped in this region is the ALPL gene (1p36.12-34), coding for the alkaline phosphatase enzyme. In a study of 66 meningiomas loss of $1 \mathrm{p}$ was strongly correlated with a reduced activity of the alkaline phosphatase enzyme ${ }^{31}$. An additional study of 54 meningiomas showed that decreased expression of alkaline phosphatase was correlated with recurrence $(p=0.0064)$ and shorter RFS $(p=0.035) .{ }^{32}$.

Although so far no chromosome 1p methylation data have been reported, some studies have examined hypermethylation of gene promotors, for example that of TP73. Hypermethylation of TP73 has been found more frequently in meningiomas with chromosome $1 \mathrm{p}$ loss $^{33}$. TP73 is suggested as potential biomarker for higher grade meningiomas, because $70-80 \%$ of high-grade tumors have TP73 promotor hypermethylation. Since the TP73 gene is included in the 1 p36 Vysis FISH probe applied in the present study, it is tempting to speculate that this biomarker may also be of value to predict meningioma regrowth, which remains to be studied ${ }^{28}$. Mutations 


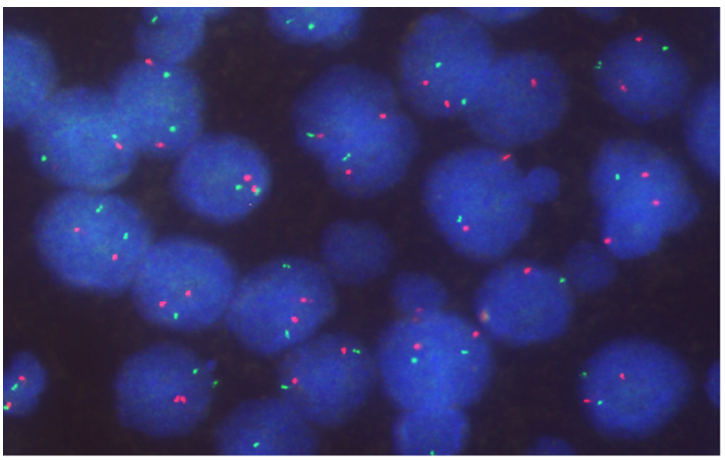

A
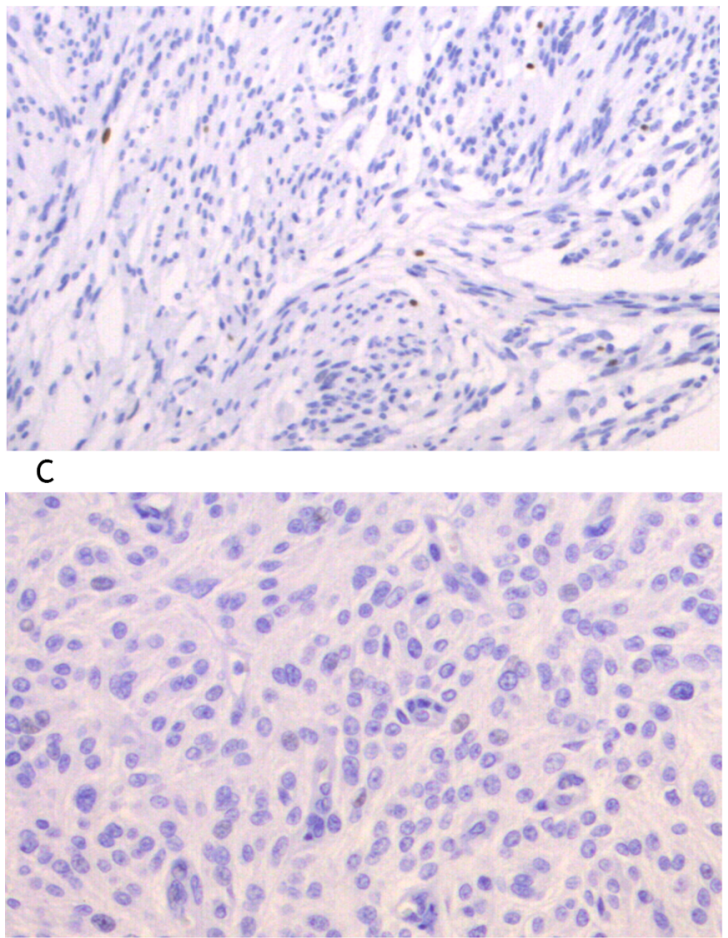

$\mathrm{E}$

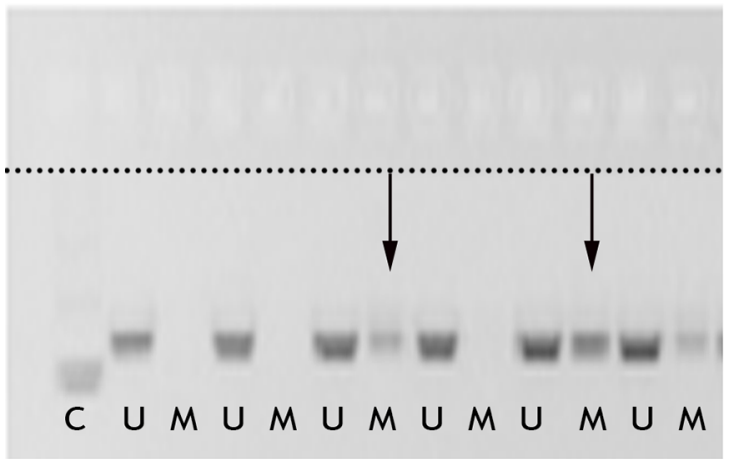

G

Figure 1. Representative examples of molecular analysis of WHO grade I meningiomas. (A-B): FISH analysis showing in (A) a tumor with two copies of 1p36 (red) and 1q25 (green) in DAPI counterstained tumor cell nuclei (blue). This tumor did not show regrowth after irradiation. In (B) arrows point to nuclei showing only one copy of 1p36 (red), indicating 1p36 loss. This tumor showed regrowth after irradiation. (C-F): Immunohistochemical analysis of Ki-67 (C-D) and NDRG4 (E-F) showing in (C) a tumor with Ki-67 expression $<1.0 \%$, (D) a tumor with Ki-67 expression $>1.0 \%$, (E) a tumor with no NDRG4 expression and in (F) a normal brain tissue control with positive NDRG4 expression. (G-H): Cropped MSP analysis of NDRG1 (G) and NDRG4 (H) (full blots in Supplementary Figs. S1-2). In (G) 2 of 6 tumors show NDRG1 hypermethylation, dashed line separates upper and lower side of the gel; compiled from 2 photographs of the gel, whereas no tumors show NDRG4 hypermethylation $(\mathbf{H})$. Abbreviations: $\mathrm{U}=$ unmethylated; $\mathrm{M}=$ methylated. $\mathrm{C}=$ unmethylated control DNA (normal lymphocytes). Magnification $100 \times(\mathbf{C}-\mathbf{D}), 200 \times(\mathbf{E}-\mathbf{F}), 400 \times(\mathbf{A}-\mathbf{B})$. 


\begin{tabular}{|l|l|l|l|l|l|}
\hline & No regrowth & Regrowth & Total & Fisher's Exact (p value) & Kaplan-Meier (p value) \\
\hline Ki-67 Expression & & & & 1 & 0.207 \\
\hline S1.0\% & $19(83 \%)$ & $4(17 \%)$ & 23 & & \\
\hline$>1.0 \%$ & $17(81 \%)$ & $4(19 \%)$ & 21 & & \\
\hline Deletion 1p36 & & & & $0.003^{*}$ & $0.001^{*}$ \\
\hline Yes & $3(38 \%)$ & $5(63 \%)$ & 8 & & \\
\hline No & $32(91 \%)$ & $3(9 \%)$ & 35 & & \\
\hline NDRG1 & & & & 0.20 & $0.046^{*}$ \\
\hline No methylation & $31(86 \%)$ & $5(14 \%)$ & 36 & & \\
\hline Methylation & $3(60 \%)$ & $2(40 \%)$ & 5 & & 0.31 \\
\hline NDRG2 & & & & 0.20 & \\
\hline No methylation & $31(86 \%)$ & $5(14 \%)$ & 36 & & - \\
\hline Methylation & $3(60 \%)$ & $2(40 \%)$ & 5 & & \\
\hline NDRG3 & & & & - & \\
\hline No methylation & $34(83 \%)$ & $7(17 \%)$ & 41 & & \\
\hline Methylation & 0 & 0 & 0 & & \\
\hline NDRG4 & & & & - & \\
\hline No methylation & $34(83 \%)$ & $7(17 \%)$ & 41 & & \\
\hline Methylation & 0 & 0 & 0 & & \\
\hline SFRP1 & & & & 1 & \\
\hline No methylation & $28(82 \%)$ & $6(18 \%)$ & 34 & & \\
\hline Methylation & $6(86 \%)$ & $1(14 \%)$ & 7 & & \\
\hline HOXA9 & & & & 1 & \\
\hline No methylation & $8(89 \%)$ & $1(11 \%)$ & 9 & & \\
\hline Methylation & $26(81 \%)$ & $6(19 \%)$ & 32 & & \\
\hline MGMT & & & & 0.69 & \\
\hline No methylation & $22(85 \%)$ & $4(15 \%)$ & 26 & & \\
\hline Methylation & $12(80 \%)$ & $3(20 \%)$ & 15 & & \\
\hline & & & & & \\
\hline
\end{tabular}

Table 2. Molecular parameters in relation to regrowth of irradiated WHO grade I meningiomas. ${ }^{*}$ Statistical significance was presumed as $p<0.05$.

in well-known cancer genes located on chromosome 1p, such as NRAS (1p13.2), GADD45A (1p31.2-31.1), CDKN2C (1p32.2), RAD45(1p32) and EPB41 (1p36.2-p34), have so far only sporadically been detected ${ }^{26,28,34,35}$.

Another interesting finding in our study is that NDRG1 hypermethylation in meningiomas with regrowth was associated with unfavorable regrowth-free survival. The NDRG family consists of four different members, namely NDRG1, 2, 3, and 4. This family of genes is involved in the regulation of cell proliferation and differentiation, and all members have specific functions ${ }^{36}$. Hypermethylation of NDRG1 has been reported to promote pathogenesis and/or proliferation of prostate, breast and gastric cancer ${ }^{37}$. A tumor suppressor function for NDRG1 has also been implicated in CNS-tumors, including neuroblastoma ${ }^{38}$ and glioma ${ }^{39}$. To our knowledge our study is the first to correlate $N D R G 1$ hypermethylation with meningioma regrowth after radiotherapy, which remains to be further studied. We could not provide evidence for NDRG2 hypermethylation as was reported by Lusis et al. as a mechanism of NDRG2 downregulation in relation to tumor recurrence ${ }^{11}$. NDRG4 is usually expressed in heart and brain cells, and has been recently found to be downregulated in colorectal cancer as a result of promotor hypermethylation ${ }^{40}$. In contrast, Kotipatruni et al. showed that NDRG4 was overexpressed in the high grade meningioma cell lines IOMM-Lee and $\mathrm{CH}-157 \mathrm{MN}^{13}$. In a subsequent study, these authors demonstrated that NDRG4 silencing inhibits proliferation and induces apoptosis in these cell lines ${ }^{14}$, suggesting an oncogenic role for NDRG4 in meningiomas. In our study NDRG4, highly expressed in all nontumoral brain control tissue, was not expressed in WHO I meningiomas. Moreover, we could not demonstrate that NDRG4 promotor hypermethylation is the possible mechanism downregulating NDRG4 expression in low grade meningiomas. A potential role for NDRG3 hypermethylation within nervous system malignancies remains uncertain as also in this study no NDRG3 promotor hypermethylation could be detected.

Although hypermethylation of $M G M T$ did not correlate with regrowth, $35 \%$ of meningioma grade I patients showed hypermethylation of MGMT, which is relatively high in comparison with $0-22 \%$ in earlier reports ${ }^{19-21}$. However, MGMT hypermethylation does not appear to be of value as an indicator for temozolomide chemotherapy in the treatment of meningiomas, because so far temozolomide has been found ineffective ${ }^{41}$.

In the current study, not all parameters related to meningioma progression have been addressed ${ }^{1}$. For example, TERT promotor mutations have been found significantly associated with recurrence with or without progression to a higher grade ${ }^{42,43}$. In this respect we could analyze 6 of our regrowth cases by digital droplet PCR which did not harbor a mutation at TERT promotor hotspot regions C228 and C250 of TERT. As a result it is unlikely that TERT promotor mutations have value in the prediction of meningioma WHO grade I regrowth after GKRS in the current study. 
A Survival Functions NDRG1 hypermethylation

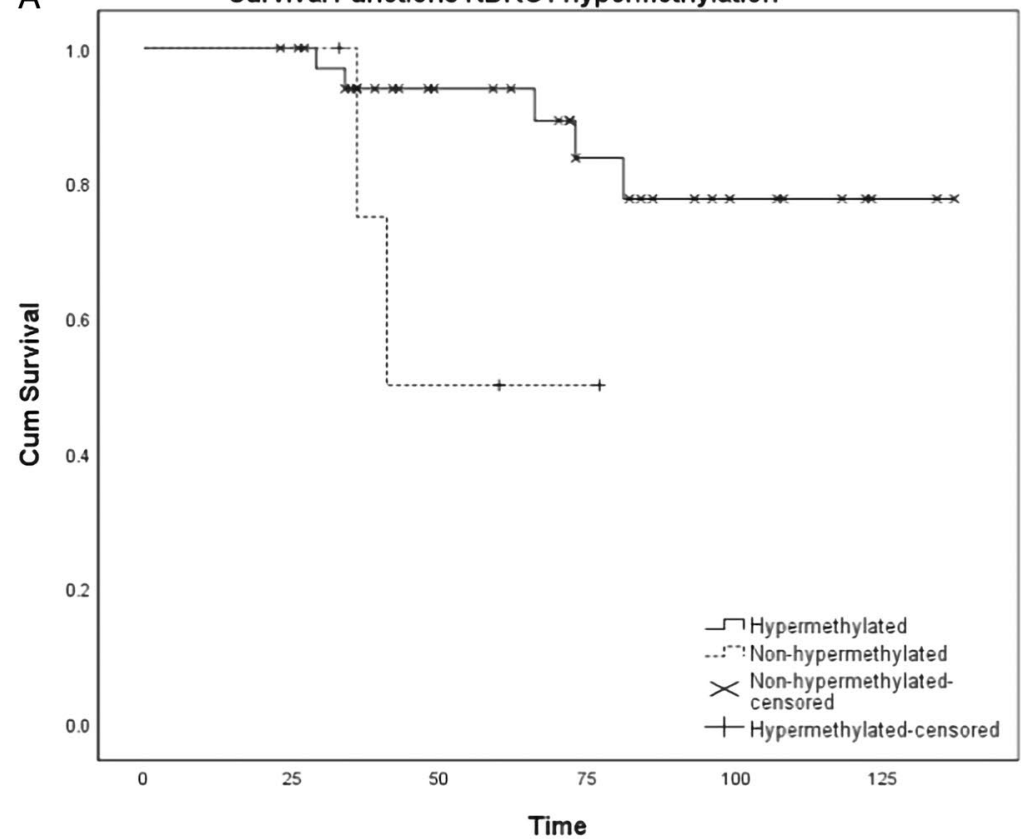

Number of patients at risk

$\begin{array}{lllllll}\text { Hypermethylated } & 4 & 4 & 2 & 1 & 0 & 0 \\ \begin{array}{l}\text { Non-hyper- } \\ \text { methylated }\end{array} & 35 & 35 & 22 & 14 & 7 & 2\end{array}$

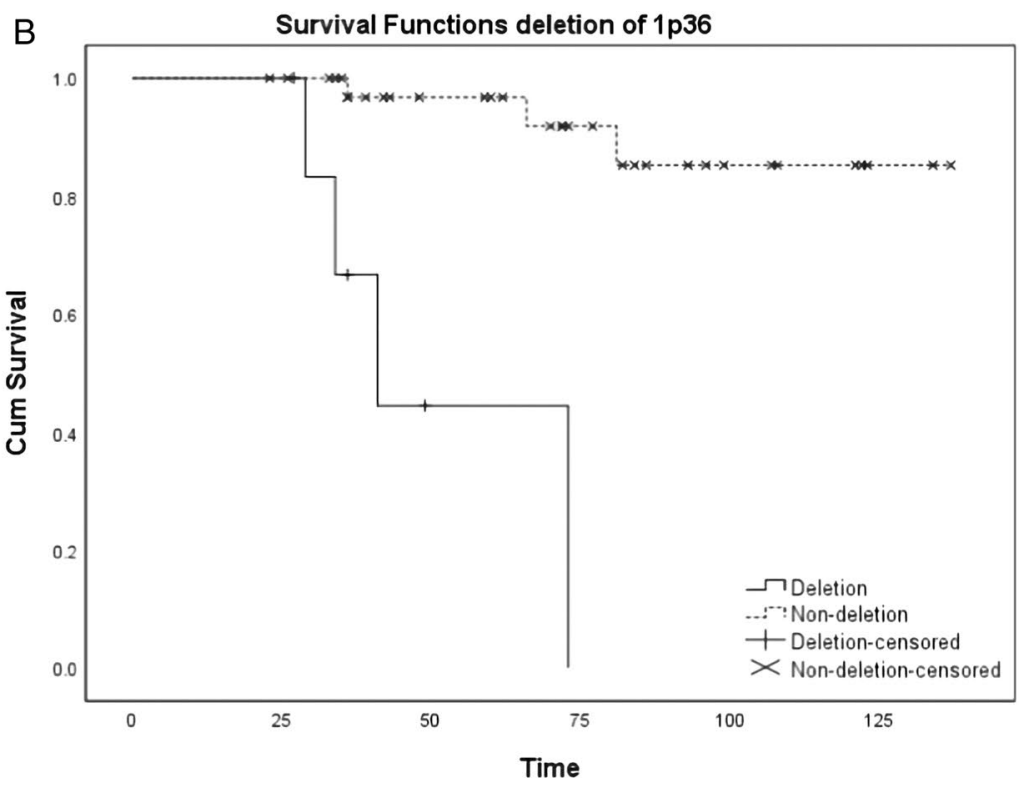

Number of patients at risk

$\begin{array}{lllllll}\text { Deletion } & 7 & 7 & 1 & 0 & 0 & 0 \\ \text { Non-deletion } & 34 & 34 & 23 & 15 & 7 & 2\end{array}$

Figure 2. Kaplan Meier survival functions of (A) NDRG1 hypermethylation and (B) deletion of 1p36 in relation to meningioma regrowth.

In contrast to some molecular markers, we did not identify clinical or radiobiological factors associated with tumor regrowth, as shown in Table 1. Dibiase et al. analyzed 139 benign meningiomas treated with GKRS and found gross tumor volume $(\mathrm{GTV})>10 \mathrm{cc}$ significantly correlated with unfavorable 5-year disease-free and 
overall survival $(p=0.038 \text { and } p=0.0001 \text {, respectively })^{44}$. We did not find a correlation between tumor volume and regrowth. The difference with our study might be explained by the fact that only $38 \%$ of their patients were treated post-operatively and no histopathologic proof of benign (WHO grade I) disease was available. Furthermore, we did not find a correlation between radiotherapy dose and regrowth. Kuhn et al. showed in a study of 279 meningiomas (21\% WHO grade I) treated with GKRS marginal doses of $\geq 12$ Gy associated with improved local control $(p=0.015)^{45}$. The difference with our study could be explained by the fact they included a less uniform group, with $20 \%$ multifocal disease, $61 \%$ unknown histology and only $43 \%$ of tumors treated with GKRS post-operatively. Besides, $63 \%$ of their local failures was classified as marginal, whereas we did not have marginal regrowth, but only in-field.

When there would be enough evidence to predict regrowth in WHO grade I meningiomas with a biomarker, the question arises how one should treat these potentially more aggressive tumors. To the best of our knowledge no studies have investigated this research question yet. One option could be to consider these tumors as progressive disease and a logical approach then would be to opt for a more frequent follow-up schedule and/ or dose escalation.

Although we have studied a uniformly postoperatively GKRS treated group of WHO grade I meningiomas, our series consisting of 44 tumors, including 8 regrowth cases, may be considered relatively small. This impacts the statistical analysis, therefore this study should be regarded as hypothesis generating. Another limitation is the relatively short follow-up time for some patients, ranging from 24 to 137 months, and the median time to regrowth in this study being 51 months. It therefore might be possible that some regrowth cases have been missed. Additional limitations of this study are its retrospective character and the fact that only Gamma Knife irradiated patients were included, whereas there are other radiotherapy modalities applied in the treatment of meningioma patients.

\section{Conclusions}

In conclusion, our results indicate that loss of 1 p36 is associated with regrowth after GKRS of WHO grade I meningiomas, which might have implications for treatment. In order to consider 1 p36 deletion as a putative indicator for regrowth, our findings need to be validated in an independent larger set of tumors.

\section{Data availability}

All data generated or analyzed during this study are included in this published article and its supplementary information files.

Received: 7 January 2021; Accepted: 21 July 2021

Published online: 12 August 2021

\section{References}

1. Preusser, M., Brastianos, P. K. \& Mawrin, C. Advances in meningioma genetics: Novel therapeutic opportunities. Nat. Rev. Neurol. 14(2), 106-115. https://doi.org/10.1038/nrneurol.2017.168 (2018).

2. Riemenschneider, M. J., Perry, A. \& Reifenberger, G. Histological classification and molecular genetics of meningiomas. Lancet Neurol. 5(12), 1045-1054. https://doi.org/10.1016/s1474-4422(06)70625-1 (2006).

3. Oya, S., Kawai, K., Nakatomi, H. \& Saito, N. Significance of Simpson grading system in modern meningioma surgery: Integration of the grade with MIB-1 labeling index as a key to predict the recurrence of WHO Grade I meningiomas. J. Neurosurg. 117(1), 121-128. https://doi.org/10.3171/2012.3.jns111945 (2012).

4. Alexiou, G. A., Gogou, P., Markoula, S. \& Kyritsis, A. P. Management of meningiomas. Clin. Neurol. Neurosurg. 112(3), $177-182$. https://doi.org/10.1016/j.clineuro.2009.12.011 (2010).

5. Kondziolka, D., Patel, A. D., Kano, H., Flickinger, J. C. \& Lunsford, L. D. Long-term outcomes after gamma knife radiosurgery for meningiomas. Am. J. Clin. Oncol. https://doi.org/10.1097/coc.0000000000000080 (2014).

6. Marciscano, A. E. et al. Benign meningiomas (WHO Grade I) with atypical histological features: Correlation of histopathological features with clinical outcomes. J. Neurosurg. 124(1), 106-114. https://doi.org/10.3171/2015.1.jns142228 (2016).

7. Hsu, D. W., Efird, J. T. \& Hedley-Whyte, E. T. MIB-1 (Ki-67) index and transforming growth factor-alpha (TGF alpha) immunoreactivity are significant prognostic predictors for meningiomas. Neuropathol. Appl. Neurobiol. 24(6), 441-452 (1998).

8. Tao, Y. et al. Clinical features and immunohistochemical expression levels of androgen, estrogen, progesterone and Ki-67 receptors in relationship with gross-total resected meningiomas relapse. Br. J. Neurosurg. 26(5), 700-704. https://doi.org/10.3109/02688697. 2012.685780 (2012).

9. Linsler, S. et al. Molecular biological determinations of meningioma progression and recurrence. PLoS ONE 9(4), e94987. https:// doi.org/10.1371/journal.pone.0094987 (2014).

10. Maillo, A. et al. Early recurrences in histologically benign/grade I meningiomas are associated with large tumors and coexistence of monosomy 14 and del(1p36) in the ancestral tumor cell clone. Neuro Oncol. 9(4), 438-446. https://doi.org/10.1215/152285172007-026 (2007).

11. Lusis, E. A. et al. Integrative genomic analysis identifies NDRG2 as a candidate tumor suppressor gene frequently inactivated in clinically aggressive meningioma. Cancer Res. 65(16), 7121-7126. https://doi.org/10.1158/0008-5472.can-05-0043 (2005).

12. Skiriute, D. et al. Tumor grade-related NDRG2 gene expression in primary and recurrent intracranial meningiomas. J. Neurooncol. 102(1), 89-94. https://doi.org/10.1007/s11060-010-0291-9(2011).

13. Kotipatruni, R. P. et al. NDRG4, the N-Myc downstream regulated gene, is important for cell survival, tumor invasion and angiogenesis in meningiomas. Integr. Biol. (Camb.) 4(10), 1185-1197. https://doi.org/10.1039/c2ib20168b (2012).

14. Kotipatruni, R. P., Ren, X., Thotala, D. \& Jaboin, J. J. NDRG4 is a novel oncogenic protein and p53 associated regulator of apoptosis in malignant meningioma cells. Oncotarget 6(19), 17594-17604. https://doi.org/10.18632/oncotarget.4009 (2015).

15. Perez-Magan, E. et al. Genetic alterations associated with progression and recurrence in meningiomas. J Neuropathol. Exp. Neurol. 71(10), 882-893. https://doi.org/10.1097/NEN.0b013e31826bf704 (2012).

16. Perez-Magan, E. et al. Differential expression profiling analyses identifies downregulation of $1 \mathrm{p}, 6 \mathrm{q}$, and $14 \mathrm{q}$ genes and overexpression of $6 \mathrm{p}$ histone cluster 1 genes as markers of recurrence in meningiomas. Neuro Oncol. 12(12), 1278-1290. https://doi.org/10. 1093/neuonc/noq081 (2010).

17. Kishida, Y. et al. Epigenetic subclassification of meningiomas based on genome-wide DNA methylation analyses. Carcinogenesis 33(2), 436-441. https://doi.org/10.1093/carcin/bgr260 (2012). 
18. Di Vinci, A. et al. HOXA7, 9, and 10 are methylation targets associated with aggressive behavior in meningiomas. Transl. Res. 160(5), 355-362. https://doi.org/10.1016/j.trsl.2012.05.007 (2012).

19. Jabini, R. et al. Pathodiagnostic parameters and evaluation of $\mathrm{O}(6)$ - methyl guanine methyl transferase gene promoter methylation in meningiomas. Gene 538(2), 348-353. https://doi.org/10.1016/j.gene.2013.12.039 (2014).

20. Bello, M. J. et al. DNA methylation of multiple promoter-associated CpG islands in meningiomas: Relationship with the allelic status at 1p and 22q. Acta Neuropathol. 108(5), 413-421. https://doi.org/10.1007/s00401-004-0911-6 (2004).

21. Aydemir, F. et al. Identification of promoter region methylation patterns of MGMT, CDKN2A, GSTP1, and THBS1 genes in intracranial meningioma patients. Genet. Test. Mol. Biomark. 16(5), 335-340. https://doi.org/10.1089/gtmb.2011.0245 (2012).

22. Bulthuis, V. J., Hanssens, P. E., Lie, S. T. \& van Overbeeke, J. J. Gamma Knife radiosurgery for intracranial meningiomas: Do we need to treat the dural tail? A single-center retrospective analysis and an overview of the literature. Surg. Neurol. Int. 5(Suppl 8), S391-S395. https://doi.org/10.4103/2152-7806.140192 (2014).

23. Derks, J. L. et al. Molecular subtypes of pulmonary large-cell neuroendocrine carcinoma predict chemotherapy treatment outcome. Clin. Cancer Res. 24(1), 33-42. https://doi.org/10.1158/1078-0432.CCR-17-1921 (2018).

24. Bulthuis VJ, Damen PJJ, Hanssens PEJ, et al. 1 p36 Loss is associated with regrowth of WHO grade I meningiomas after gamma knife radiosurgery. J. Neurol. Surg. B. 2016;77(S 02):LFP-07-04. https://doi.org/10.1055/s-0036-1592589

25. Weber, R. G. et al. Analysis of genomic alterations in benign, atypical, and anaplastic meningiomas: Toward a genetic model of meningioma progression. Proc. Natl. Acad. Sci. U. S. A. 94(26), 14719-14724. https://doi.org/10.1073/pnas.94.26.14719 (1997).

26. Boström, J. et al. Alterations of the tumor suppressor genes CDKN2A (p16(INK4a)), p14(ARF), CDKN2B (p15(INK4b)), and CDKN2C (p18(INK4c)) in atypical and anaplastic meningiomas. Am. J. Pathol. 159(2), 661-669. https://doi.org/10.1016/S00029440(10)61737-3 (2001).

27. Twijnstra, A., Claessen, S. M. H., Blaauw, G., Janzer, R. \& Speel, E. J. M. Comparative genomic hybridization analysis of primary and recurrent meningiomas. Neurology. 62(5), A546 (2004).

28. Suppiah, S. et al. Molecular and translational advances in meningiomas. Neuro Oncol. 21(Supplement_1), 4-17. https://doi.org/ 10.1093/neuonc/noy178 (2019).

29. Bi, W. L. et al. Genomic landscape of high-grade meningiomas. NPJ Genomic Med. 2(1), 15. https://doi.org/10.1038/s41525-0170014-7 (2017).

30. Tabernero, M. D. et al. Delineation of commonly deleted chromosomal regions in meningiomas by high-density single nucleotide polymorphism genotyping arrays. Genes Chromosomes Cancer. 51(6), 606-617. https://doi.org/10.1002/gcc.21948 (2012).

31. Niedermayer, I. et al. Loss of alkaline phosphatase activity in meningiomas: A rapid histochemical technique indicating progression-associated deletion of a putative tumor suppressor gene on the distal part of the short arm of chromosome 1. J. Neuropathol. Exp. Neurol. 56(8), 879-886 (1997).

32. Bouvier, C. et al. Lack of alkaline phosphatase activity predicts meningioma recurrence. Am. J. Clin. Pathol. 124(2), 252-258. https://doi.org/10.1309/482A-WA3U-8DJU-EXWU (2005).

33. Lomas, J. et al. Methylation status of TP73 in meningiomas. Cancer Genet. Cytogenet. 148(2), 148-151 (2004).

34. Piaskowski, S. et al. GADD45A and EPB41 as tumor suppressor genes in meningioma pathogenesis. Cancer Genet Cytogenet. 162(1), 63-67. https://doi.org/10.1016/j.cancergencyto.2005.02.009 (2005).

35. Joachim, T. et al. Comparative analysis of the NF2, TP53, PTEN, KRAS, NRAS and HRAS genes in sporadic and radiation-induced human meningiomas. Int. J. Cancer. 94(2), 218-221 (2001).

36. Vaes, N. et al. A combined literature and in silico analysis enlightens the role of the NDRG family in the gut. Biochim. Biophys. Acta Gen. Subj. 1862(10), 2140-2151. https://doi.org/10.1016/j.bbagen.2018.07.004 (2018).

37. Li, Y., Pan, P., Qiao, P. \& Liu, R. Downregulation of N-myc downstream regulated gene 1 caused by the methylation of CpG islands of NDRG1 promoter promotes proliferation and invasion of prostate cancer cells. Int. J. Oncol. 47(3), 1001-1008. https://doi.org/ 10.3892/ijo.2015.3086 (2015).

38. Matsushita, K. et al. Low NDRG1 mRNA expression predicts a poor prognosis in neuroblastoma patients. Pediatr. Surg. Int. 29(4), 363-368. https://doi.org/10.1007/s00383-012-3248-6 (2013).

39. Blaes, J. et al. NDRG1 prognosticates the natural course of disease in WHO grade II glioma. J. Neurooncol. 117(1), 25-32. https:// doi.org/10.1007/s11060-013-1357-2 (2014).

40. Melotte, V. et al. N-Myc downstream-regulated gene 4 (NDRG4): A candidate tumor suppressor gene and potential biomarker for colorectal cancer. J. Natl. Cancer Inst. 101(13), 916-927. https://doi.org/10.1093/jnci/djp131 (2009).

41. Nigim, F., Wakimoto, H., Kasper, E., Ackermans, L. \& Temel, Y. Emerging medical treatments for meningioma in the molecular era. Biomedicines. 6(3), 86. https://doi.org/10.3390/biomedicines6030086 (2018).

42. Sahm, F. et al. TERT promoter mutations and risk of recurrence in meningioma. J. Natl. Cancer Inst. https://doi.org/10.1093/jnci/ djv377 (2016).

43. Goutagny, S. et al. High incidence of activating TERT promoter mutations in meningiomas undergoing malignant progression. Brain Pathol. 24(2), 184-189. https://doi.org/10.1111/bpa.12110 (2014).

44. DiBiase, S. J. et al. Factors predicting local tumor control after gamma knife stereotactic radiosurgery for benign intracranial meningiomas. Int. J. Radiat. Oncol. Biol. Phys. 60(5), 1515-1519. https://doi.org/10.1016/j.ijrobp.2004.05.073 (2004).

45. Kuhn, E. N. et al. Patterns of recurrence after stereotactic radiosurgery for treatment of meningiomas. Neurosurg Focus. 35(6), E14. https://doi.org/10.3171/2013.8.focus13283 (2013).

\section{Acknowledgements}

We thank J.J. van Overbeeke for fruitful discussion, and S. Stevens-Timmermans for technical support.

\section{Author contributions}

Conceptualization, V.B. and E.J.S.; Methodology, V.B. and E.J.S.; Validation, P.D., V.B. and E.J.S.; Formal Analysis, P.D. and V.B.; Investigation, P.D. and V.B.; Resources, P.D., V.B., P.H., S.T.L., R.F., V.M., K.W., A.R., J.B. and E.J.S.; Data Curation, P.D. and V.B.; Writing_Original Draft Preparation, P.D., V.B. and E.J.S.; Writing-Review \& Editing, P.D., V.B., P.H., S.T.L., R.F., V.M., K.W., A.R., J.B. and E.J.S.; Visualization, P.D., V.B. and E.J.S.; Supervision, V.B. and E.J.S.; Project Administration, V.B. and E.J.S.

\section{Competing interests}

The authors declare no competing interests.

\section{Additional information}

Supplementary Information The online version contains supplementary material available at https://doi.org/ 10.1038/s41598-021-95956-x.

Correspondence and requests for materials should be addressed to E.J.M.S. 
Reprints and permissions information is available at www.nature.com/reprints.

Publisher's note Springer Nature remains neutral with regard to jurisdictional claims in published maps and institutional affiliations.

(c) (i) Open Access This article is licensed under a Creative Commons Attribution 4.0 International License, which permits use, sharing, adaptation, distribution and reproduction in any medium or format, as long as you give appropriate credit to the original author(s) and the source, provide a link to the Creative Commons licence, and indicate if changes were made. The images or other third party material in this article are included in the article's Creative Commons licence, unless indicated otherwise in a credit line to the material. If material is not included in the article's Creative Commons licence and your intended use is not permitted by statutory regulation or exceeds the permitted use, you will need to obtain permission directly from the copyright holder. To view a copy of this licence, visit http://creativecommons.org/licenses/by/4.0/.

(C) The Author(s) 2021 